\title{
Weakly exchange rings whose units are sums of two idempotents
}

\section{P. V. Danchev}

Institute of Mathematics and Informatics, Bulgarian Academy of Sciences, Acad. G. Bonchev str., block 8, 1113 Sofia, Bulgaria

For citation: Danchev P. V. Weakly exchange rings whose units are sums of two idempotents. Vestnik of Saint Petersburg University. Mathematics. Mechanics. Astronomy, 2019, vol. 6 (64), issue 2, pp. 265-269. https://doi.org/10.21638/11701/spbu01.2019.208

We prove that if every element in the unit group $U(R)$ of a weakly exchange ring $R$ is a sum of two idempotents of $R$, then every element in the center $C(R)$ of $R$ is a sum of two central idempotents of $R$. This somewhat enlarges results due to Koşan - Ying - Zhou published in Can. Math. Bull. (2016) as well as due to Karimi - Koşan - Zhou published in Contemp. Math. (2018). Moreover, we show that each nilpotent of order not exceeding 2 in a von Neumann regular ring is a difference of two special (left-right symmetric) idempotents. This somewhat refines a recent result by O'Meara stated in a still unpublished preprint (2018).

Keywords: weakly exchange rings, units, idempotents, center.

1. Introduction and Background. Everywhere in the text of the present paper, all our rings $R$ are assumed to be associative, containing the identity element 1, which in general differs from the zero element 0 of $R$, and all subrings are unital (i. e. containing the same identity as that of the former ring). Our terminology and notations are mainly in agreement with [1]. For instance, $J(R)$ denotes the Jacobson radical of $R, U(R)$ the set of all units in $R, I d(R)$ the set of all idempotents in $R, N i l(R)$ the set of all nilpotents in $R$, and $C(R)$ the center of $R$. We shall also denote the set of all unipotents (that are sums of 1 and a nilpotent) in $R$ like $\operatorname{Unip}(R)$, that is, $\operatorname{Unip}(R)=1+\operatorname{Nil}(R)$.

Recall that a ring $R$ is said to be $\pi$-regular if for every $r \in R$ there exist an element $a$ from $R$ and a positive integer $i$, both depending on $r$, such that $r^{i}=r^{i} a r^{i}$. In particular, when $i=1$ for each $r$, the ring $R$ is known as von Neumann regular. An important subclass of the class of $\pi$-regular rings forms the class of so-termed strongly $\pi$-regular rings for which, in terms above, $r^{i}=r^{2 i} a$. Besides, a proper subclass of the class of von Neumann regular rings forms the class of so-called unit-regular rings for which, in the current terms, $r=\operatorname{rar}$ with $a \in U(R)$. It is principally known that any von Neumann regular element of a strongly $\pi$-regular ring is unit-regular (compare with the comments on p. 641 from [2]). However, a von Neumann regular nilpotent element in general may not be unit-regular (see also [2] for more details). Nevertheless, it was established in [2, Theorem 4] that a nilpotent element each power of which is von Neumann regular must be unit-regular; thus, any nilpotent in a von Neumann regular ring is necessarily unit-regular. We shall freely use this nice fact below with proper citation.

We also recall that a ring $R$ is called exchange provided for any $r \in R$ the existence of $e \in r R \cap I d(R)$ with $1-e \in(1-r) R$, and weakly exchange provided for any $r \in R$

(c) Санкт-Петербургский государственный университет, 2019 
the existence of $e \in r R \cap I d(R)$ with $1-e \in(1-r) R \cup(1+r) R$. It is well known that $\pi$-regular rings themselves are exchange.

Answering a question due to the present author, it was shown in [3] that in strongly $\pi$-regular rings (and more generally, even in the classical von Neumann regular rings) the inclusion $\operatorname{Nil}(R) \subseteq I d(R)-I d(R)$, and hence the inclusion $\operatorname{Unip}(R) \subseteq I d(R)+I d(R)$, always holds; notice that we shall somewhat refine this in the rather specific case of nilpotent elements of order not exceeding 2. Since $\operatorname{Unip}(R) \subseteq U(R)$, we are motivated to study the structure of those rings $R$ for which $U(R) \subseteq I d(R)+I d(R)$. Specifically, we will treat the partial converse of whether or not (weakly) exchange rings whose units are sums of two idempotents are $\pi$-regular, as well as of whether or not rings of characteristic 2 containing only unipotent units and whose elements are sums of two idempotents are $\pi$-regular (compare with the two questions posed at the end of the article). About the latter query, it is worth noticing that in [4] exchange rings $R$ with $U(R)=U n i p(R)$ were completely described as those rings for which $J(R)$ is contained in $N i l(R)$ and the quotient $R / J(R)$ is a Boolean ring.

2. Main Results. First and foremost, we begin with the promised refining of the mentioned above result from [3]. To that aim, we shall say that two idempotents $e, f$ of a given ring are left-right symmetric, provided the two equalities $e f=e$ and $f e=f$ are valid. Our statement is closely related to the so-called "idempotent sum number" explored in detail in [5].

So, we come to the following expected refinement.

Proposition 2.1. In von Neumann regular rings every nilpotent of exponent less than or equal to 2 is the difference of two left-right symmetric idempotents.

Proof. Let $q$ be a nilpotent in a von Neumann regular ring $R$ such that $q^{2}=0$. Referring to [2, Theorem 4], $q$ has to be a unit-regular element. Then, $q$ can be represented as $q=u e$ for some $u \in U(R)$ and $e \in I d(R)$. Hence, ueue $=0$ implies that $e u e=0$ which, in turn, yields $e q=0$ and $q e=q$. One easily verifies that the presentation of $q$ like

$$
q=[e-e q(1-e)]-[e-(1-e) q e]
$$

is fulfilled, where the first bracket is obviously equal to $e$ whereas the second bracket is equal to $e-q$, say $f$. Certainly, both $e$ and $f$ are idempotents as well as an easy check shows that $e f=e(e-q)=e-e q=e$ and $f e=(e-q) e=e-q e=e-q=f$, as claimed.

However, it is worthwhile noticing that, what we have proved in this statement could not have happened looking at the proof of the corresponding result in [3]. Likewise, it is not obvious at all whether this fact can be considerably extended to arbitrary nilpotents of exponent exceeding 2. It is done in [3] for arbitrary nilpotents, but, unfortunately, the proof there does not directly guarantee that the existing idempotents in the difference are left-right symmetric.

The following technicality extends a corresponding result from [6] as well as our proof somewhat strengthens that in [7, Lemma 3.11].

Lemma 2.2. In a ring $R$ for which $U(R) \subseteq I d(R)+I d(R)$, the decomposition $R \cong R_{1} \times R_{2}$ is valid, where $R_{1}$ is a ring with $2=0$ and $R_{2}$ is a ring with $3=0$. In particular, $6=0$ in $R$. 
Proof. Write $-1=e+f$ for some $e, f \in I d(R)$, and hence $-1-e=f$. By squaring, we get that $1+3 e=-1-e$, i. e. $2=-4 e$. Squaring again, we obtain that $4=16 e=-8$. Finally, $12=4 \times 3=0$. Since $\operatorname{gcd}(4,3)=1$, with the classical Chinese Remainder Theorem at hand, one decomposes $R \cong R_{1} \times R_{2}$ for some rings $R_{1}$ and $R_{2}$ whose unit groups continue to be sums of two idempotent sets of $R_{1}$ and $R_{2}$, respectively, and for which $4=0$ in $R_{1}$ and $3=0$ in $R_{2}$. We claim even that $2=0$ is fulfilled in $R_{1}$. In fact, since $2^{2}=0$ holds in $R_{1}$, it follows that $2 \in N i l\left(R_{1}\right)$. Thus, $3 \in U\left(R_{1}\right)$ and, thereby, by assumption it must be that $2=g-h$ for some two $g, h \in \operatorname{Id}\left(R_{1}\right)$. Hence, $1-g=-1-h$ and squaring this, we get that $1-g=1+3 h=1-h=-1-h$ as $4=0$. Finally, the last equality yields that $2=0$, as claimed. The conclusion that $6=0$ holds in $R$ is now immediate.

We now come to the folklore omnibus needed for our successful presentation.

Proposition 2.3. Let $R$ be a weakly exchange ring. Then $C(R) \subseteq U(R) \pm I d(R)$.

Proof. Given an arbitrary element $x \in C(R)$, there exists an idempotent $e \in x R$ such that $1-e \in(1-x) R$ or $1-e \in(1+x) R$. So, we write that $e=x c=c x$ for some $c \in R$. Letting $c x c=c^{2} x=x c^{2}$, we verify that $e=x a=a x$ as well as that $c e=a e=a$. First, assume that $1-e$ lies in $(1+x) R$, so one may write that $1-e=(1+x) d=d(1+x)$. Setting $b=d(1+x) d=d^{2}(1+x)=(1+x) d^{2}$, we obtain that $1-e=(1+x) b=b(1+x)$. Seeing that $b=b(1-e)=d(1+x) d(1-e)=d(1-e)=d(1+x) d$, one derives now that $[x+(1-e)][a+b]=1$ because $(1-e) a=e b=0$. By analogy, $[a+b][x+(1-e)]=1$ since $a(1-e)=b e=0$. Finally, one infers that $x+(1-e)$ is a unit, as required.

Next, we can process similarly for the situation when $1-e \in(1-x) R$ to get that $x-(1-e)$ is a unit with the inverse $a-b$, thus giving up the wanted assertion after all.

Let us recollect now that a ring is said to be tripotent if each of its elements satisfies the equation $x^{3}=x$. So, tripotent rings are obviously commutative von Neumann regular rings.

The next technical statement is crucial.

Lemma 2.4. Suppose that $R$ is a ring of characteristic 3 such that $t=v+f$ for some $t, v, f \in R$ with $t^{2}=0, v^{2}=1$ and $f^{2}=f$. Then $f=1$.

Proof. Squaring the equality $t=v+f$, we derive that $1+f+v f+f v=0$. Multiplying this by $f$ on both sides, we obtain that $2 f+v f+f v f=0=2 f+f v+f v f$. Hence $v f=f v$ and thus $1+f=-2 v f=v f$ as $3=0$. Multiplying this by $f$ again on the right, we get that $2 f=v f$, so that $f=-v f$ because $3=0$. This, in turn, yields that $0=1+f-v f=1+2 f=1-f$ since $3=0$, i. e. $f=1$, as required.

The next comments shed some more light on the previous technicality.

Remark 2.5. Actually, substituting $f=1$ in the expression for $t$, one detects that $t=v+1$ and, hence, by squaring it follows that $v=2$ as $3=0$. Therefore, $t=0-$ compare with the corresponding third paragraph of the proof of Theorem 2.6 (see below).

We now have all the ingredients necessary to establish the following chief statement.

Theorem 2.6. Suppose $R$ is a weakly exchange ring such that $U(R) \subseteq I d(R)+$ $I d(R)$. Then $C(R)=I d(C(R))+I d(C(R))$. In particular, $C(R)$ is a tripotent ring. 
Proof. According to Lemma 2.2, one decomposes that $R \cong R_{1} \times R_{2}$ for some weakly exchange rings $R_{1}$ and $R_{2}$ such that $2=0$ in $R_{1}, 3=0$ in $R_{2}$ and such that $U\left(R_{1}\right) \subseteq I d\left(R_{1}\right)+\operatorname{Id}\left(R_{1}\right), U\left(R_{2}\right) \subseteq I d\left(R_{2}\right)+\operatorname{Id}\left(R_{2}\right)$.

We first assert that $C\left(R_{1}\right)$ is a Boolean ring. To this goal, we firstly note that, as $2=0$ in $R_{1}$, it is an exchange ring with $C\left(R_{1}\right) \subseteq U\left(R_{1}\right)+\operatorname{Id}\left(R_{1}\right)$ and $U\left(R_{1}\right) \subseteq \operatorname{Id}\left(R_{1}\right)+\operatorname{Id}\left(R_{1}\right)$ by an appeal to Proposition 2.3. Therefore, $C\left(R_{1}\right) \subseteq \operatorname{Id}\left(R_{1}\right)+\operatorname{Id}\left(R_{1}\right)+\operatorname{Id}\left(R_{1}\right)$ and so $C\left(R_{1}\right) \subseteq I d\left(R_{1}\right)$. In fact, for every $c \in C\left(R_{1}\right)$ we write that $c=e+f+h$ for $e, f, h \in \operatorname{Id}\left(R_{1}\right)$. Observing that $(e+f) h=h(e+f)$ and squaring $c=e+f+h$, we deduce that $c^{2}=(e+f)^{2}+h^{2}=c+e f+f e$. But $c^{2}-c \in C\left(R_{1}\right)$ whence $c^{2}-c=e f+f e$ ensures that $\left(c^{2}-c\right) e=e f e+f e=e f e+e f=e\left(c^{2}-c\right)$ and, thus, that $e f=f e$. Finally, $c^{2}-c=2 e f=0$ deriving that $c^{2}=c$, as asserted.

We now claim that the second direct factor $R_{2}$ is a reduced ring and, thus, it is abelian. To show this, for any $a_{2} \in R_{2}$ with $a_{2}^{2}=0$, we write that $-1+a_{2}=e_{2}+f_{2}$ for some $e_{2}, f_{2} \in I d\left(R_{2}\right)$ because $-1+a_{2} \in U\left(R_{2}\right)$. Thus, $a_{2}=\left(1+e_{2}\right)+f_{2}$, where $\left(1+e_{2}\right)^{2}=1$ since $3=0$. Owing to Lemma 2.4 , we get that $f_{2}=1$ and so $1-e_{2}=$ $-a_{2} \in \operatorname{Id}\left(R_{2}\right) \cap \operatorname{Nil}\left(R_{2}\right)=\{0\}$. Finally, $a_{2}=0$, as claimed. Furthermore, in accordance with [8], we conclude that $R_{2}$ must be weakly clean, that is, $R_{2}=U\left(R_{2}\right) \pm \operatorname{Id}\left(R_{2}\right)$ which allows us to detect that $R_{2}=I d\left(R_{2}\right)+I d\left(R_{2}\right) \pm I d\left(R_{2}\right)$. This enables us that $R_{2}$ should be even commutative, that is, $R_{2}=C\left(R_{2}\right)$ and consequently $r_{2}^{3}=r_{2}$ for all $r_{2} \in R_{2}$. Thus, as it is well-known, $R_{2}$ is a subdirect product of a family of copies of the field $\mathbb{Z}_{3}$.

Further, since $C(R) \cong C\left(R_{1}\right) \times C\left(R_{2}\right)=C\left(R_{1}\right) \times R_{2}$, one concludes that all elements of the ring $C(R)$ satisfy the equation $x^{3}=x$ and, thereby, $C(R)$ is too a subdirect product of a single copy or isomorphic copies of $\mathbb{Z}_{3}$, as needed. This substantiates our initial statement after all.

Some more commentaries in that way are worthwhile as well.

Remark 2.7. Concerning the explicit structure of the ring $R$, we discover the status of clean (which are necessarily exchange) rings $R$, that is, any element in it is the sum of a unit and an idempotent, whose units are sums of two idempotents. One then observes that these rings $R$ are of the type $R=I d(R)+I d(R)+I d(R)$. In the abelian case these rings are of necessity commutative and have a complete description (see [9]), that unambiguously shows that they have to be $\pi$-regular.

Moreover, it is well known that the center of a non-abelian (weakly) exchange ring need not be exchange (e.g. see [10]) and thus it is not von Neumann regular either. That is why, the condition stated on $U(R)$ is essential and cannot be ignored. However, the center of a von Neumann regular ring is strongly regular and so it is a clean ring.

We finish off our work with the following two queries of some interest and importance.

Problem 2.8. Is it true that (weakly) exchange rings whose units are sums of two idempotents are $\pi$-regular (and, in some partial cases, even von Neumann regular) rings?

Problem 2.9. Suppose that $R=\operatorname{Id}(R)+\operatorname{Id}(R)$ is a ring of characteristic 2 whose $U(R)=\operatorname{Unip}(R)$. Does it follow that $R$ is $\pi$-regular and, in particular, even exchange?

In these two aspects, at first glance, we could consider the commutative case and especially the indecomposable commutative ring $\mathbb{Z}_{4}=\{0,1,2,3 \mid 4=0\}$ which is manifestly not von Neumann regular, but in which the following relationships hold: $J\left(\mathbb{Z}_{4}\right)=$ $\operatorname{Nil}\left(\mathbb{Z}_{4}\right)=\{0,2\}, I d\left(\mathbb{Z}_{4}\right)=\{0,1\}, U\left(\mathbb{Z}_{4}\right)=\operatorname{Unip}\left(\mathbb{Z}_{4}\right)=\{1,3\}$ and $\mathbb{Z}_{4} / J\left(\mathbb{Z}_{4}\right) \cong \mathbb{Z}_{2}$. 
Besides, it is easily seen that the unit 3 cannot be represented as a sum of two idempotents; however, it is the difference of two idempotents like $3=0-1$. This means that the condition $U(R) \subseteq I d(R)+I d(R)$ in Theorem 2.6 cannot be replaced by the more general one $U(R) \subseteq I d(R) \pm I d(R)$.

Nevertheless, it seems that the solution to the latter problem stated above will be in general "no", provided that a ring $R$ having the following properties could be successfully constructed: $R=I d(R)+I d(R) \neq I d(R), 2=0, J(R)=\{0\}$ and $U(R)=U n i p(R) \neq$ $\{1\}$. In fact, if these rings $R$ were exchange, appealing to [4] it must be that $R$ is Boolean, contrary to the construction.

\section{References}

1. Lam T.Y., A First Course in Noncommutative Rings, in Graduate Texts in Math. 131 (second edition, Springer-Verlag, Berlin - Heidelberg - New York, 2001). (2016).

2. Khurana D., "Unit-regularity of regular nilpotent elements", Algebr. \& Repres. Th. 19, 641-644

3. O'Meara K., "Nilpotents often the difference of two idempotents" (draft privately circulated on March 2018).

4. Danchev P. V., Lam T. Y., "Rings with unipotent units", Publ. Math. Debrecen 88, 449-466 (2016).

5. Danchev P. V., Nasibi E., "The idempotent sum number and $n$-thin unital rings", Ann. Univ. Sci. Budapest (Sect. Math.) 59, 85-98 (2016).

6. Koşan T., Ying Z., Zhou Y., "Rings in which every element is a sum of two tripotents", Can. Math. Bull. 59, 661-672 (2016).

7. Karimi-Mansoub A., Koşan M. T., Zhou Y., "Rings in which every unit is a sum of a nilpotent and an idempotent", Contemp. Math. 715, 189-203 (2018).

8. Chin A. Y. M., Qua K. T., "A note on weakly clean rings", Acta Math. Hungar. 132, 113-116 (2011).

9. Danchev P. V., "Rings whose elements are sums of three or differences of two commuting idempotents", Bull. Iran. Math. Soc. 44, 1641-1651 (2018).

10. Burgess W. D., Raphael R., "On embedding rings in clean rings", Commun. Algebra 41, 552-564 (2013).

Received: August 13, 2018

Accepted: December 20, 2018

Author's information:

PeterV.Danchev-PhD; danchev@math.bas.bg, pvdanchev@yahoo.com 